\title{
The Flow and Heat Transfer of a Nanofluid Past a Stretching/Shrinking Sheet with a Convective Boundary Condition
}

\author{
Syahira Mansur' ${ }^{1}$ and Anuar Ishak ${ }^{2}$ \\ ${ }^{1}$ Department of Mathematics and Statistics, Faculty of Science, Technology and Human Development, \\ Universiti Tun Hussein Onn Malaysia, Batu Pahat, 86400 Parit Raja, Johor, Malaysia \\ ${ }^{2}$ School of Mathematical Sciences, Faculty of Science and Technology, Universiti Kebangsaan Malaysia (UKM), \\ 43600 Bangi, Selangor, Malaysia
}

Correspondence should be addressed to Anuar Ishak; anuarishak@yahoo.com

Received 26 June 2013; Revised 2 September 2013; Accepted 3 September 2013

Academic Editor: Jianzhong Lin

Copyright (C) 2013 S. Mansur and A. Ishak. This is an open access article distributed under the Creative Commons Attribution License, which permits unrestricted use, distribution, and reproduction in any medium, provided the original work is properly cited.

The boundary layer flow of a nanofluid past a stretching/shrinking sheet with a convective boundary condition is studied. Numerical solutions to the governing equations are obtained using a shooting method. The results are found for the local Nusselt number and the local Sherwood number as well as the temperature and concentration profiles for some values of the convective parameter, stretching/shrinking parameter, Brownian motion parameter, and thermophoresis parameter. The results indicate that the local Nusselt number is consistently higher for higher values of the convective parameter. However, the local Nusselt number decreases with increasing values of the Brownian motion parameter as well as the thermophoresis parameter. In addition, the local Sherwood number increases with increasing Brownian motion parameter and decreases with increasing convective parameter and thermophoresis parameter.

\section{Introduction}

The boundary layer flow over a stretching sheet is important in applications such as extrusion, wire drawing, metal spinning, and hot rolling [1]. Crane [2] pioneered the study of stretching sheet by presenting an exact analytical solution for the steady two-dimensional stretching of a plate in a quiescent fluid. Since then, many authors have considered various aspects of this problem. Wang [3] extended Crane's study to include both suction and slip effects at the boundary. Sahoo [4] considered non-Newtonian fluid in his research.

Recently, the study on the flow over a shrinking sheet has garnered considerable attention. Miklavčič and Wang [5] initiated the study of flow over a shrinking sheet. They found that the vorticity is not confined within a boundary layer, and a steady flow cannot exist without exerting adequate suction at the boundary. Ever since, numerous studies emerge, investigating different aspects of this problem. Fang et al. [6] used a second-order slip flow in their research. Bhattacharyya et al. [7] analyzed the effects of partial slip on laminar boundary layer stagnation-point flow and heat transfer towards a shrinking sheet.

Inclusion of nanoparticles into the base fluid such as water is known to increase the heat transfer capability of the fluid. Choi and Eastman [8] discovered that the addition of less than $1 \%$ of nanoparticles into the base fluid doubles the heat conductivity of the fluid. Other characteristics of nanofluid include minimal clogging of tube and long term stability as compared to other fluids containing micro- and millimeter sized particles (see [9-13]). Two models have been constantly used by researchers to study the behaviour of nanofluid, namely, the Tiwari-Das model [14] and Buongiorno model [15]. Contrary to the Tiwari-Das model [14] that focuses on volumetric fraction of nanoparticles, Buongiorno model [15] pays more attention to Brownian motion 
and thermophoresis effects. Furthermore, instead of focusing on the thermophysical properties of the nanofluid, Buongiorno model shifted the focus to explaining the further heat transfer enhancement observed in convective situations. Buongiorno model was used in many recent papers, for example, Nield and Kuznetsov [16-18], Kuznetsov and Neild $[19,20]$, Khan and Pop [21], Bachok et al. [22, 23], and Khan and Aziz [24], among others.

In the boundary layer flow and heat transfer analysis, constant surface temperature and heat flux are customarily used. However, there are times when heat transfer at the surface relies on the surface temperature, as what mostly occurs in heat exchangers. In this situation, convective boundary condition is used to replace the condition of prescribed surface temperature or prescribed surface heat flux. Aziz [25] employed the convective boundary condition in his research to study the heat transfer characteristics for the Blasius flow. Ishak [26] introduced the effects of suction and injection at the boundary. Makinde and Aziz [27] investigated the boundary layer flow of a nanofluid past a stretching sheet with a convective surface boundary condition.

Motivated by the above-mentioned investigations and applications, we extend the study of Makinde and Aziz [27] to include both stretching and shrinking cases, in addition to the suction effect. Although there are many studies conducted on the shrinking or stretching sheet, little work has been done to include both the stretching and shrinking cases. For the shrinking case which was not considered by Makinde and Aziz [27], the solutions do not exist since vorticity could not be confined within the boundary layer. However, with an added suction effect to confine the vorticity, the solution may exist. The dependency of the local Nusselt number and the local Sherwood number on four parameters, namely, the stretching/shrinking, convective, Brownian motion, and thermophoresis parameters, is the main focus of the present investigation. Numerical solutions are presented graphically and in tabular forms to show the effects of these parameters on the local Nusselt number and the local Sherwood number.

\section{Mathematical Formulation}

Consider a steady, two-dimensional $(x, y)$ boundary layer flow of a viscous and incompressible fluid over a stretching/shrinking sheet immersed in a nanofluid. It is assumed that the stretching/shrinking velocity is in the form $U_{w}=$ $a x$, where $a$ is a positive constant and $x$ is the coordinate measured along the stretching/shrinking surface. It is also assumed that the constant mass flux velocity is $v_{0}$ with $v_{0}<$ 0 for suction and $v_{0}>0$ for injection or withdrawal of the fluid. The nanofluid is confined to $y>0$, where $y$ is the coordinate measured normal to the stretching/shrinking surface, as shown in Figure 1. It is further assumed that the bottom surface of the sheet is heated by convection from a hot fluid at temperature $T_{f}$ which provides a heat transfer coefficient $h$. The surface temperature $T_{w}$ is the result of a convective heating process characterized by the hot fluid.
The governing equations for the steady conservation of mass, momentum, thermal energy, and nanoparticle volume fraction equations can be written as [15-24]

$$
\begin{gathered}
\frac{\partial u}{\partial x}+\frac{\partial v}{\partial y}=0 \\
u \frac{\partial u}{\partial x}+v \frac{\partial u}{\partial y}=-\frac{1}{\rho_{f}} \frac{\partial P}{\partial x}+\nu\left(\frac{\partial^{2} u}{\partial x^{2}}+\frac{\partial^{2} u}{\partial y^{2}}\right) \\
u \frac{\partial v}{\partial x}+v \frac{\partial v}{\partial y}=-\frac{1}{\rho_{f}} \frac{\partial P}{\partial y}+v\left(\frac{\partial^{2} v}{\partial x^{2}}+\frac{\partial^{2} v}{\partial y^{2}}\right) \\
u \frac{\partial T}{\partial x}+v \frac{\partial T}{\partial y}=\alpha\left(\frac{\partial^{2} T}{\partial x^{2}}+\frac{\partial^{2} T}{\partial y^{2}}\right) \\
+\tau\left\{D_{B}\left(\frac{\partial \varphi}{\partial x} \frac{\partial T}{\partial x}+\frac{\partial \varphi}{\partial y} \frac{\partial T}{\partial y}\right)\right. \\
\left.+\frac{D_{T}}{T_{\infty}}\left[\left(\frac{\partial T}{\partial x}\right)^{2}+\left(\frac{\partial T}{\partial y}\right)^{2}\right]\right\} \\
u \frac{\partial \varphi}{\partial x}+v \frac{\partial \varphi}{\partial y}=D_{B}\left(\frac{\partial^{2} \varphi}{\partial x^{2}}+\frac{\partial^{2} \varphi}{\partial y^{2}}\right)+\frac{D_{T}}{T_{\infty}}\left(\frac{\partial^{2} T}{\partial x^{2}}+\frac{\partial^{2} T}{\partial y^{2}}\right)
\end{gathered}
$$

where $u$ and $v$ are the velocity components along the $x$ and $y$-axis, respectively, $P$ is the fluid pressure, $T$ is the fluid temperature, $\alpha$ is the thermal diffusivity, $v$ is the kinematic viscosity, $D_{B}$ is the Brownian diffusion coefficient, $D_{T}$ is the thermophoresis diffusion coefficient, and $\varphi$ is the nanoparticle volume fraction. Furthermore, $\tau=(\rho c)_{p} /(\rho c)_{f}$ is the ratio between the effective heat capacity of the fluid with $\rho_{f}$ and $\rho_{p}$ being the density of the fluid and the density of the particles, respectively, and $c_{f}$ and $c_{p}$ denote the specific heat of the fluid and the particle at constant pressure, respectively. The subscript $\infty$ represents the values at large values of $y$ (outside the boundary layer). Details of the derivation of (4) and (5) are given in the papers by Buongiorno [15] and Nield and Kuznetsov [17].

Equations (1)-(5) are subjected to the following boundary conditions [25-27]:

$$
\begin{gathered}
v=v_{0}, \quad u=\sigma U_{w}(x), \\
-k \frac{\partial T}{\partial y}=h\left(T_{f}-T\right), \quad \varphi=\varphi_{w} \quad \text { at } y=0, \\
u \longrightarrow 0, \quad v \longrightarrow 0, \quad T \longrightarrow T_{\infty}, \\
\varphi \longrightarrow \varphi_{\infty} \quad \text { as } y \longrightarrow \infty,
\end{gathered}
$$

where $\sigma$ is a constant with $\sigma>0$ for stretching and $\sigma<$ 0 for shrinking and $k$ is the thermal conductivity of the base fluid. The subscript $w$ denotes the values at the solid surface. The governing equations (1)-(5) subjected to the 


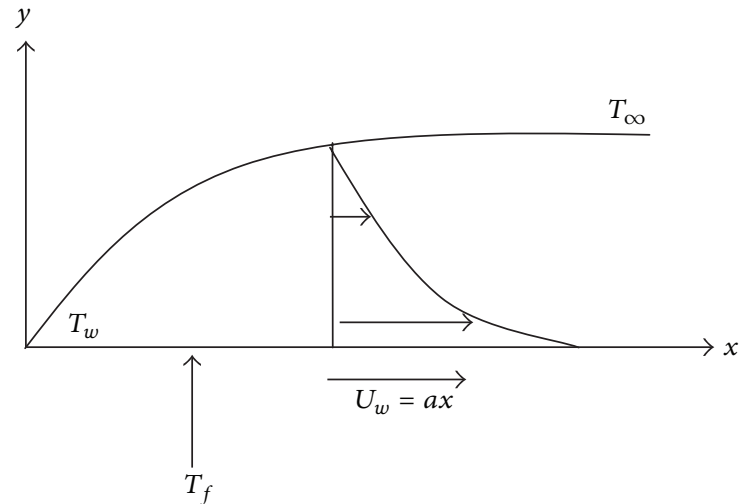

(a)

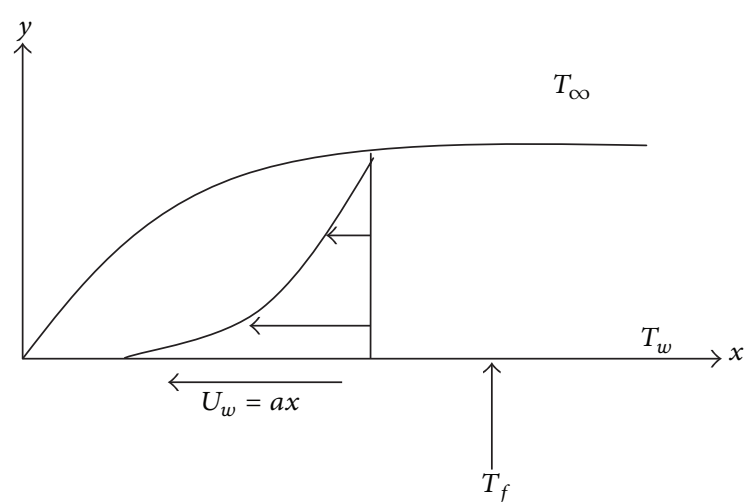

(b)

FIGURE 1: Geometry of the problem for (a) stretching and (b) shrinking sheets.

boundary conditions (6) can be expressed in a simpler form by introducing the following transformation:

$$
\begin{aligned}
& \psi=\left(U_{w} \nu x\right)^{1 / 2} f(\eta), \quad \eta=\left(\frac{U_{w}}{\nu x}\right)^{1 / 2} y, \\
& \theta(\eta)=\frac{T-T_{\infty}}{T_{f}-T_{\infty}}, \quad \beta(\eta)=\frac{\varphi-\varphi_{\infty}}{\varphi_{w}-\varphi_{\infty}}
\end{aligned}
$$

where $\eta$ is the similarity variable and $\psi$ is the stream function defined as $u=\partial \psi / \partial y$ and $v=-\partial \psi / \partial x$, which identically satisfies (1). By employing the boundary layer approximations and the similarity variables (7), (2)-(5) reduce to the following nonlinear ordinary differential equations:

$$
\begin{gathered}
f^{\prime \prime \prime}+f f^{\prime \prime}-f^{\prime 2}=0, \\
\frac{1}{\operatorname{Pr}} \theta^{\prime \prime}+f \theta^{\prime}+\mathrm{Nb} \beta^{\prime} \theta^{\prime}+\mathrm{N} t \theta^{\prime 2}=0, \\
\beta^{\prime \prime}+\frac{\mathrm{Nt}}{\mathrm{Nb}} \theta^{\prime \prime}+\operatorname{Le} f \beta^{\prime}=0,
\end{gathered}
$$

and the boundary conditions (6) become

$$
\begin{gathered}
f(0)=S, \quad f^{\prime}(0)=\sigma, \\
\theta^{\prime}(0)=-\gamma[1-\theta(0)], \quad \beta(0)=1, \\
f^{\prime}=0, \quad \theta=0, \quad \beta=0 \quad \text { as } \eta \longrightarrow \infty,
\end{gathered}
$$

where primes denote differentiation with respect to $\eta$. Further, $\mathrm{Pr}$ is the Prandtl number, $\mathrm{Nb}$ is the Brownian motion parameter, $\mathrm{Nt}$ is the thermophoresis parameter, Le is the Lewis number, $S$ is the mass flux parameter with $S>0$ for suction and $S<0$ for injection, and $\gamma$ is the Biot number (convective parameter), which are defined as

$$
\begin{gathered}
\operatorname{Pr}=\frac{\nu}{\alpha}, \quad \mathrm{Nb}=\frac{\tau D_{B}\left(\varphi_{w}-\varphi_{\infty}\right)}{\nu}, \\
\mathrm{Nt}=\frac{\tau D_{T}\left(T_{w}-T_{\infty}\right)}{\nu T_{\infty}}, \\
\mathrm{Le}=\frac{\nu}{D_{B}}, \quad S=-\frac{v_{0}}{\sqrt{a v}}, \quad \gamma=\frac{h}{k} \sqrt{\frac{\nu}{a} .}
\end{gathered}
$$

When $\mathrm{Nb}=\mathrm{Nt}=0$, the present problem reduces to a regular viscous fluid, and the nanoparticle volume fraction equation (10) becomes ill-posed and is of no physical significance.

The physical quantities of interest are the skin friction coefficient $C_{f}$, the local Nusselt number $\mathrm{Nu}_{x}$, and the local Sherwood number $\mathrm{Sh}_{x}$ which are defined as

$$
\begin{gathered}
C_{f}=\frac{\tau_{w}}{\rho U_{w}^{2}}, \quad \mathrm{Nu}_{x}=\frac{x q_{w}}{k\left(T_{f}-T_{\infty}\right)}, \\
\mathrm{Sh}_{x}=\frac{x q_{m}}{D_{B}\left(\varphi_{w}-\varphi_{\infty}\right)},
\end{gathered}
$$

where $\tau_{w}, q_{w}$, and $q_{m}$ are the surface shear stress, the heat, and mass fluxes, respectively, which are given by [23]

$$
\begin{gathered}
\tau_{w}=\mu\left(\frac{\partial u}{\partial y}\right)_{y=0}, \quad q_{w}=-k\left(\frac{\partial T}{\partial y}\right)_{y=0}, \\
q_{m}=-D_{B}\left(\frac{\partial \varphi}{\partial y}\right)_{y=0} .
\end{gathered}
$$

Using the similarity variables (7), we obtain

$$
\begin{aligned}
C_{f} \operatorname{Re}_{x}^{1 / 2}= & f^{\prime \prime}(0), \quad \mathrm{Nu}_{x} \mathrm{Re}_{x}^{-1 / 2}=-\theta^{\prime}(0), \\
& \mathrm{Sh}_{x} \mathrm{Re}_{x}^{-1 / 2}=-\beta^{\prime}(0),
\end{aligned}
$$

where $\operatorname{Re}_{x}=U_{w} x / \nu$ is the local Reynolds number. 


\section{Results and Discussion}

The set of ordinary differential equations (8)-(10) with the boundary conditions (11) and (12) were solved numerically using a shooting method. In this method, the dual solutions are obtained by setting different initial guesses for the values of $f^{\prime \prime}(0),-\theta^{\prime}(0)$, and $-\beta^{\prime}(0)$, where all profiles satisfy the far field boundary conditions (12) asymptotically but with different boundary layer thicknesses. The problem for a regular (viscous) fluid involves five parameters: Prandtl number, stretching/shrinking, suction/injection, and convective parameters. In this study, three parameters are added, namely, the Lewis number, Brownian motion, and thermophoresis parameters. The asymptotic boundary conditions (12) at $\eta=$ $\infty$ are replaced by $\eta=15$ as customary in the boundary layer analysis. This choice is adequate for the velocity, temperature, and concentration profiles to reach the far field boundary conditions asymptotically. We note that when $\sigma=1$, the closed-form solution for (8) was reported by P. S. Gupta and A. S. Gupta [28] and Ishak et al. [29], which is given by $f(\eta)=$ $\zeta-(1 / \zeta) e^{-\zeta \eta}$, where $\zeta-(1 / \zeta)=S$ and $\zeta>0$. Consequently, this expression leads to $f^{\prime \prime}(\eta)=-\zeta e^{-\zeta \eta}$. Numerical results for $f^{\prime \prime}(0)$ are compared with the analytical results obtained and shown in Table 1 where they are found to be in excellent agreement, cementing the validity of the numerical results in this study. From Table 1, it is obvious that the skin friction coefficient $f^{\prime \prime}(0)$ decreases with the increasing of $\sigma$.

Variations with $\sigma$ of the local Nusselt number $\mathrm{Nu}_{x} \mathrm{Re}_{x}^{-1 / 2}$ (heat transfer rates) and the local Sherwood number $\mathrm{Sh}_{x} \mathrm{Re}_{x}^{-1 / 2}$ (concentration rates) for different values of parameters are presented in Figures 2-7. As can be seen in these figures, there are more than one solution obtained for a fixed value of $\sigma$. When $\sigma$ is equal to a certain value $\sigma=\sigma_{c}$ where $\sigma_{c}(<0)$ is the critical value of $\sigma$, there is only one solution, and when $\sigma<\sigma_{c}$, there is no solution. From these figures, it is seen that the values of $\left|\sigma_{c}\right|$ remain unchanged for different values of $\gamma, \mathrm{Nt}$, and $\mathrm{Nb}$. This is clear from (8)-(12) where the thermal field does not affect the flow field. However, based on our computations, we found that by increasing the mass flux parameter $S$, the range of solutions widens where $\sigma_{c}=-1.0000,-1.5625$, and -2.2500 for $S=2,2.5$, and 3 , respectively.

From Figures 2-7, the local Nusselt number and the local Sherwood number for a nanofluid change with the variations of $\gamma, \mathrm{Nt}$, and $\mathrm{Nb}$. It can be seen that the surface heat transfer from Figures 2-4 generally decreases as $\sigma$ increases. From these figures, it is shown that the local Nusselt number (Figure 2) is consistently higher for a nanofluid with higher values of convective parameter $\gamma$. As $\gamma$ is directly proportional to the heat transfer coefficient $h$, it is inversely proportional to the thermal resistance [25]. Thus, as $\gamma$ increases, the heat resistance decreases and hence increases the heat transfer rate at the surface. Figures 3 and 4 show the effects of thermophoresis and Brownian motion parameter ( $\mathrm{Nt}$ and $\mathrm{Nb}$, resp.) on the local Nusselt number. The figures show that by increasing both parameters, the heat transfer rate at the surface reduces. To further test this observation, we construct Tables 2(a) and 3(a) where we compute variations of the local
TABLE 1: Values of $f^{\prime \prime}(0)$ for different values of $S$.

\begin{tabular}{|c|c|c|c|}
\hline$S$ & $\sigma$ & $\begin{array}{c}\qquad f^{\prime \prime}(0) \\
\text { P. S. Gupta and A. S. Gupta } \\
{[28]} \\
\text { Ishak et al. [29] }\end{array}$ & Present study \\
\hline \multirow{5}{*}{2} & -0.2 & - & 0.3789 \\
\hline & -0.1 & - & 0.1949 \\
\hline & 0.1 & - & -0.2049 \\
\hline & 0.5 & - & -1.1124 \\
\hline & 1 & -2.4142 & -2.4142 \\
\hline \multirow{5}{*}{2.5} & -0.2 & - & 0.4834 \\
\hline & -0.1 & - & 0.2489 \\
\hline & 0.1 & - & -0.2539 \\
\hline & 0.5 & - & -1.3431 \\
\hline & 1 & -2.8508 & -2.8508 \\
\hline \multirow{5}{*}{3} & -0.2 & - & 0.5864 \\
\hline & -0.1 & - & 0.2966 \\
\hline & 0.1 & - & -0.3033 \\
\hline & 0.5 & - & -1.5792 \\
\hline & 1 & -3.3028 & -3.3028 \\
\hline
\end{tabular}

Nusselt number with $\mathrm{Nt}$ and $\mathrm{Nb}$, and $\mathrm{Nt}$ and $\gamma$. Again, we can see that the surface heat transfer is lower for higher values of $\mathrm{Nt}$ and $\mathrm{Nb}$. These results concur with previous results obtained by Nield and Kuznetsov (see [16-18]). Increasing Brownian motion and thermophoresis parameters causes the thermal boundary layer to thicken, thus decreasing the local Nusselt number. This phenomenon is explained by Rasekh et al. [30] where they claimed that the increased Brownian motion impacts a larger extent of the fluid and the thermophoresis diffusion penetrates deeper into the fluid. On the other hand, to further attest to the results shown in Figure 2, Table 3(a) shows that for every value of Nt, the local Nusselt number increases with the increment of $\gamma$. From Figures 3 and 4 and Table 2(a), it is interesting to note that while the local Nusselt number changes by approximately $0.4 \%$ when $\mathrm{Nb}$ is increased, the local Nusselt number decreases by only $0.006 \%$ when Nt is increased. From this observation, it seems that Brownian motion parameter $\mathrm{Nb}$ affects the surface heat transfer more than the thermophoresis parameter $\mathrm{Nt}$ does.

As opposed to the local Nusselt number, the local Sherwood number increases with increasing $\sigma$. However, Figure 5 shows that increasing $\gamma$ does not favour mass exchange efficiency, and thus the local Sherwood number drops. Table 3(b) supports this claim where we compute the variations of the local Sherwood number with $\mathrm{Nt}$ and $\gamma$. Figures 6 and 7 depict the variations of mass transfer rates with different $\mathrm{Nt}$ and $\mathrm{Nb}$. Similar to the local Nusselt number, increasing the thermophoresis parameter $\mathrm{Nt}$ will cause the local Sherwood number to drop. Nevertheless, the latter increases with increasing $\mathrm{Nb}$. These observations are also shown in Table 2(b). From the table, it is noted that although the increment of $\mathrm{Nb}$ increases the local Sherwood number, higher values of $\mathrm{Nt}$ lower the mass transfer rates. Through 
TABLE 2: (a) Variation of the local Nusselt number $\mathrm{Nu}_{x}$ with $\mathrm{Nb}$ and $\mathrm{Nt}$ and (b) variation of the local Sherwood number $\mathrm{Sh}_{x}$ with $\mathrm{Nb}$ and $\mathrm{Nt}$ for $\gamma=0.1$, Le $=2$, and $\operatorname{Pr}=6.8$.

(a)

\begin{tabular}{ccccccccrr}
\hline & $\mathrm{Nb}=0.1$ & \multicolumn{2}{c}{$\mathrm{Nb}=0.2$} & \multicolumn{2}{c}{$\mathrm{Nb}=0.3$} & \multicolumn{2}{c}{$\mathrm{Nb}=0.4$} & \multicolumn{2}{c}{$\mathrm{Nb}=0.5$} \\
$\mathrm{Nt}$ & $\mathrm{Nu}_{x}$ & $\mathrm{Nt}$ & $\mathrm{Nu}_{x}$ & $\mathrm{Nt}$ & $\mathrm{Nu}_{x}$ & $\mathrm{Nt}$ & $\mathrm{Nu}_{x}$ & $\mathrm{Nt}$ & $\mathrm{Nu}_{x}$ \\
\hline 0.1 & 0.099140 & 0.1 & 0.098973 & 0.1 & 0.098752 & 0.1 & 0.098453 & 0.1 & 0.098043 \\
0.2 & 0.099139 & 0.2 & 0.098972 & 0.2 & 0.098750 & 0.2 & 0.098451 & 0.2 & 0.098039 \\
0.3 & 0.099138 & 0.3 & 0.098971 & 0.3 & 0.098749 & 0.3 & 0.098448 & 0.3 & 0.098035 \\
0.4 & 0.099137 & 0.4 & 0.098970 & 0.4 & 0.098747 & 0.4 & 0.098446 & 0.4 & 0.098031 \\
0.5 & 0.099136 & 0.5 & 0.098969 & 0.5 & 0.098746 & 0.5 & 0.098443 & 0.5 & 0.098026 \\
\hline
\end{tabular}

(b)

\begin{tabular}{cccccccccc}
\hline \multicolumn{2}{c}{$\mathrm{Nb}=0.1$} & \multicolumn{2}{c}{$\mathrm{Nb}=0.2$} & \multicolumn{2}{c}{$\mathrm{Nb}=0.3$} & \multicolumn{2}{c}{$\mathrm{Nb}=0.4$} & \multicolumn{2}{c}{$\mathrm{Nb}=0.5$} \\
$\mathrm{Nt}$ & $\mathrm{Sh}_{x}$ & $\mathrm{Nt}$ & $\mathrm{Sh}_{x}$ & $\mathrm{Nt}$ & $\mathrm{Sh}_{x}$ & $\mathrm{Nt}$ & $\mathrm{Sh}_{x}$ & $\mathrm{Nt}$ & $\mathrm{Sh}_{x}$ \\
\hline 0.1 & 3.866697 & 0.1 & 3.916454 & 0.1 & 3.933054 & 0.1 & 3.941369 & 0.1 & 3.946375 \\
0.2 & 3.767307 & 0.2 & 3.866821 & 0.2 & 3.900021 & 0.2 & 3.916650 & 0.2 & 3.926663 \\
0.3 & 3.667917 & 0.3 & 3.817189 & 0.3 & 3.866988 & 0.3 & 3.891933 & 0.3 & 3.906953 \\
0.4 & 3.568529 & 0.4 & 3.767557 & 0.4 & 3.833956 & 0.4 & 3.867217 & 0.4 & 3.887244 \\
0.5 & 3.469141 & 0.5 & 3.717926 & 0.5 & 3.800925 & 0.5 & 3.842501 & 0.5 & 3.867536 \\
\hline
\end{tabular}

TABLE 3: (a) Variation of the local Nusselt number $\mathrm{Nu}_{x}$ with $\gamma$ and $\mathrm{Nt}$ and (b) variation of the local Sherwood number $\mathrm{Sh}_{x}$ with $\gamma$ and $\mathrm{Nt}$ for $\mathrm{Nb}=0.5, \mathrm{Le}=2$, and $\mathrm{Pr}=6.8$.

(a)

\begin{tabular}{lccccccccc}
\hline \multicolumn{2}{c}{$\mathrm{Nt}=0.1$} & \multicolumn{2}{c}{$\mathrm{Nt}=0.2$} & \multicolumn{2}{c}{$\mathrm{Nt}=0.3$} & \multicolumn{2}{c}{$\mathrm{Nt}=0.4$} & \multicolumn{2}{c}{$\mathrm{Nt}=0.5$} \\
$\gamma$ & $\mathrm{Nu}_{x}$ & $\gamma$ & $\mathrm{Nu}_{x}$ & $\gamma$ & $\mathrm{Nu}_{x}$ & $\gamma$ & $\mathrm{Nu}_{x}$ & $\gamma$ & $\mathrm{Nu}_{x}$ \\
\hline 0.1 & 0.098043 & 0.1 & 0.098039 & 0.1 & 0.098035 & 0.1 & 0.098031 & 0.1 & 0.098026 \\
0.2 & 0.192308 & 0.2 & 0.192276 & 0.2 & 0.192243 & 0.2 & 0.192211 & 0.2 & 0.192178 \\
0.3 & 0.282986 & 0.3 & 0.282884 & 0.3 & 0.282779 & 0.3 & 0.282673 & 0.3 & 0.282565 \\
0.4 & 0.370261 & 0.4 & 0.370029 & 0.4 & 0.369792 & 0.4 & 0.369549 & 0.4 & 0.369301 \\
0.5 & 0.454302 & 0.5 & 0.453870 & 0.5 & 0.453427 & 0.5 & 0.452972 & 0.5 & 0.452503 \\
\hline
\end{tabular}

(b)

\begin{tabular}{rccccccccc}
\hline \multicolumn{2}{c}{$\mathrm{Nt}=0.1$} & \multicolumn{2}{c}{$\mathrm{Nt}=0.2$} & \multicolumn{2}{c}{$\mathrm{Nt}=0.3$} & \multicolumn{2}{c}{$\mathrm{Nt}=0.4$} & \multicolumn{2}{c}{$\mathrm{Nt}=0.5$} \\
$\gamma$ & $\mathrm{Sh}_{x}$ & $\gamma$ & $\mathrm{Sh}_{x}$ & $\gamma$ & $\mathrm{Sh}_{x}$ & $\gamma$ & $\mathrm{Sh}_{x}$ & $\gamma$ & \multicolumn{2}{c}{$\mathrm{Sh}_{x}$} \\
\hline 0.1 & 3.946375 & 0.1 & 3.926663 & 0.1 & 3.906953 & 0.1 & 3.887244 & 0.1 & 3.867536 \\
0.2 & 3.927421 & 0.2 & 3.888766 & 0.2 & 3.850122 & 0.2 & 3.811491 & 0.2 & 3.772871 \\
0.3 & 3.909188 & 0.3 & 3.852327 & 0.3 & 3.795505 & 0.3 & 3.738723 & 0.3 & 3.681982 \\
0.4 & 3.891640 & 0.4 & 3.817279 & 0.4 & 3.743010 & 0.4 & 3.668834 & 0.4 & 3.594756 \\
0.5 & 3.874741 & 0.5 & 3.783559 & 0.5 & 3.692549 & 0.5 & 3.601719 & 0.5 & 3.511676 \\
\hline
\end{tabular}

Figures 5-7, it is interesting to see that the change occurring in the mass transfer rates is almost monotonous for all values of Nt. However, the difference in the local Sherwood number increases as $\gamma$ increases and decreases as $\mathrm{Nb}$ increases.

Figures 8-11 show the samples of temperature and concentration profiles for different values of $\gamma$ and Nt. These profiles satisfy the far field boundary conditions (12) asymptotically which support the validity of the numerical results obtained, as well as supporting the existence of the dual solutions shown in Figures 2-7. For a similar problem where dual solutions exist, Merkin [31], Weidman et al. [32], and Postelnicu and Pop [33] have shown that the first solution is linearly stable and physically realizable, while the second solution is not. Thus for the present problem, it is expected that only the first solution is physically relevant. The generated temperature profiles shown in Figure 8 are qualitatively similar to those of Aziz [25] and Ishak [26]. From the figure, it is seen that the temperature increases as $\gamma$ increases. As mentioned earlier, the surface temperature $T_{w}$ depends on the convective parameter $\gamma$. As $\gamma$ reaches $\infty$, the surface temperature will approach 1 , which conforms to the boundary condition (11). Figure 9 shows the temperature profiles for different values of $\mathrm{Nt}$. It is observed that the change in temperature occurs only slightly for every change in $\mathrm{Nt}$. This phenomenon supports the results obtained in Figure 3 and Table 2(a). Figures 8 and 9 agree with the observation of 


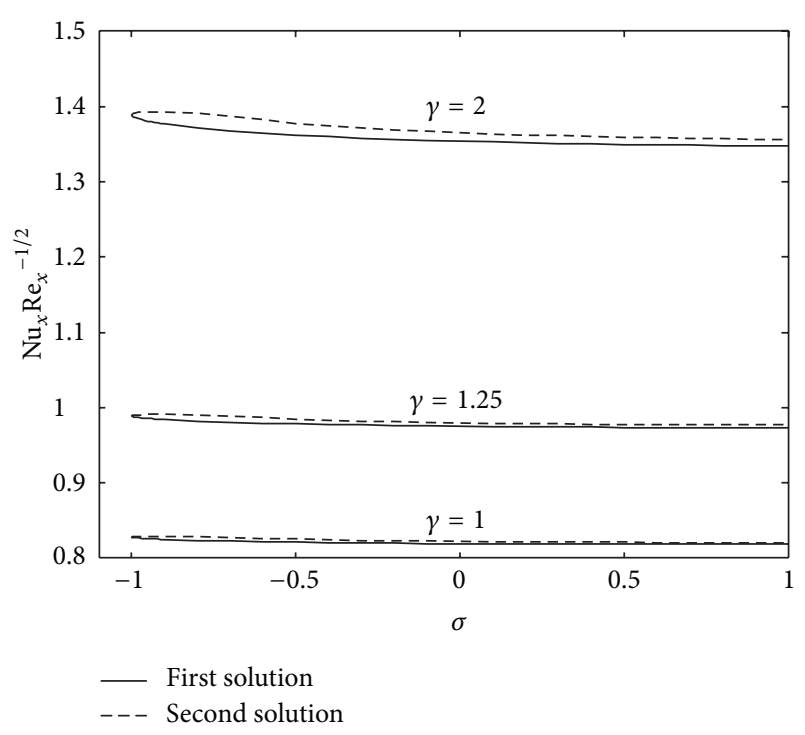

Figure 2: Variations of the local Nusselt number (heat transfer rate) with $\sigma$ for different values of $\gamma$ when $\mathrm{Nb}=\mathrm{Nt}=0.5, \mathrm{Le}=2, \mathrm{Pr}=6.8$, and $S=2$.

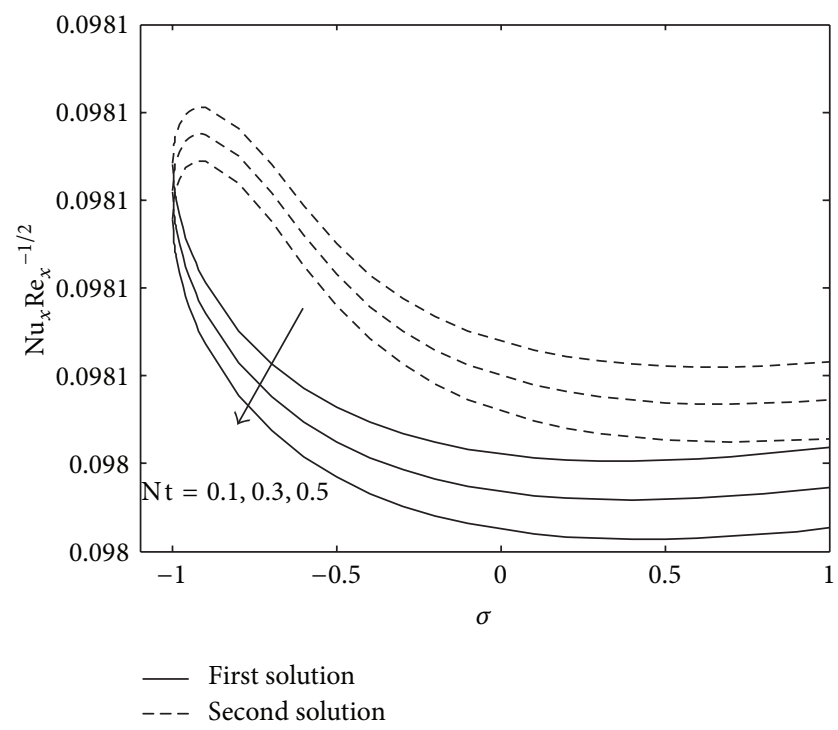

FIgURE 3: Variations of the local Nusselt number (heat transfer rate) with $\sigma$ for different values of $\mathrm{Nt}$ when $\mathrm{Nb}=0.5, \mathrm{Le}=2, \operatorname{Pr}=6.8, \gamma=$ 0.1 , and $S=2$.

Makinde and Aziz [27] where the thermal boundary layer thickens with the rise in temperature as convective parameter and thermophoresis parameter intensify.

The concentration profiles corresponding to the temperature profiles in Figures 8 and 9 are shown in Figures 10 and 11. In Figures 8 and 9, it is observed that the temperature increases as the convective parameter $\gamma$ and thermophoresis parameter Nt increase. Due to the dependency of the concentration on the temperature field, we expect that higher convective and thermophoresis parameters would allow a deeper penetration of the concentration [27]. Hence, it is seen

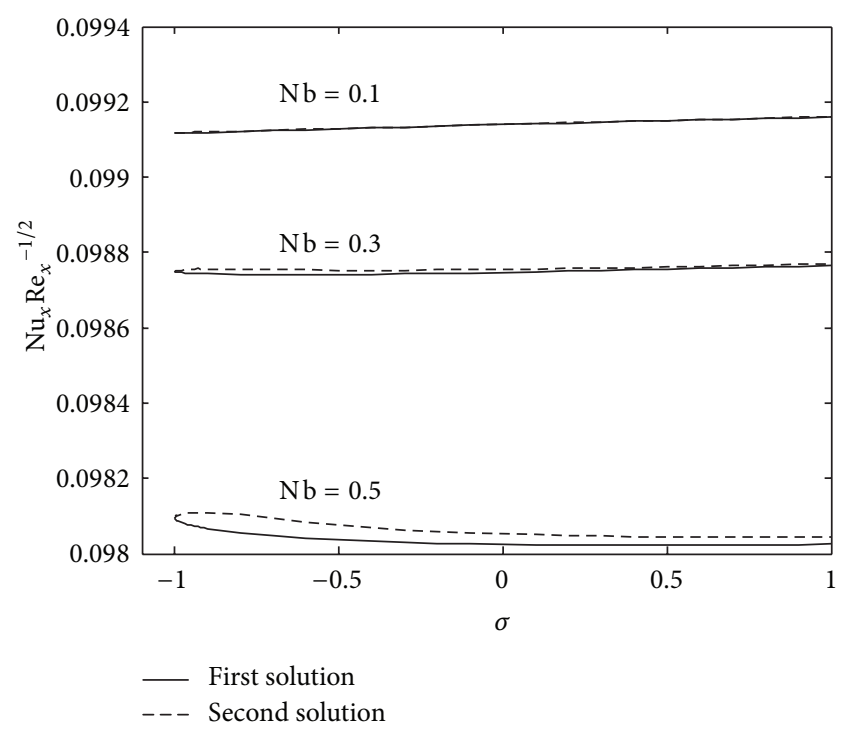

FIGURE 4: Variations of the local Nusselt number (heat transfer rate) with $\sigma$ for different values of $\mathrm{Nb}$ when $\mathrm{Nt}=0.5, \mathrm{Le}=2, \mathrm{Pr}=6.8, \gamma=$ 0.1 , and $S=2$

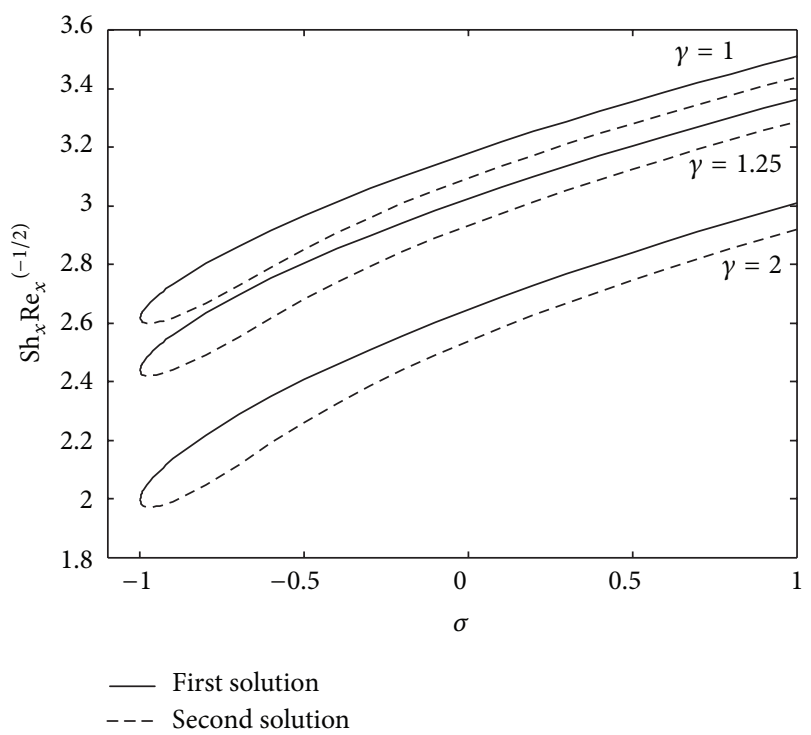

FIgURE 5: Variations of the local Sherwood number (concentration rates) with $\sigma$ for different values of $\gamma$ when $\mathrm{Nb}=\mathrm{Nt}=0.5, \mathrm{Le}=2, \mathrm{Pr}$ $=6.8$, and $S=2$.

that in Figures 10 and 11, the concentration increases with the increasing of convective parameter $\gamma$ and thermophoresis parameter Nt.

\section{Conclusions}

The boundary layer flow of a nanofluid past a stretching/shrinking sheet with a convective boundary condition was studied. The effects of stretching/shrinking parameter, convective parameter, Brownian motion parameter and thermophoresis parameter on the local Nusselt number and local 


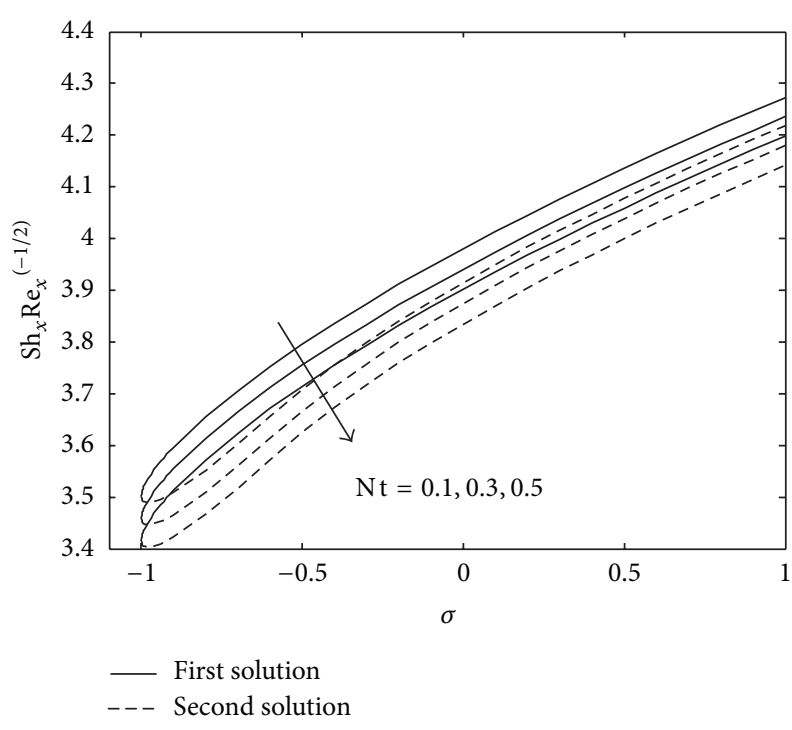

FIGURE 6: Variations of the local Sherwood number (concentration rates) with $\sigma$ for different values of $\mathrm{Nt}$ when $\mathrm{Nb}=0.5$, $\mathrm{Le}=2, \mathrm{Pr}=$ $6.8, \gamma=0.1$, and $S=2$.

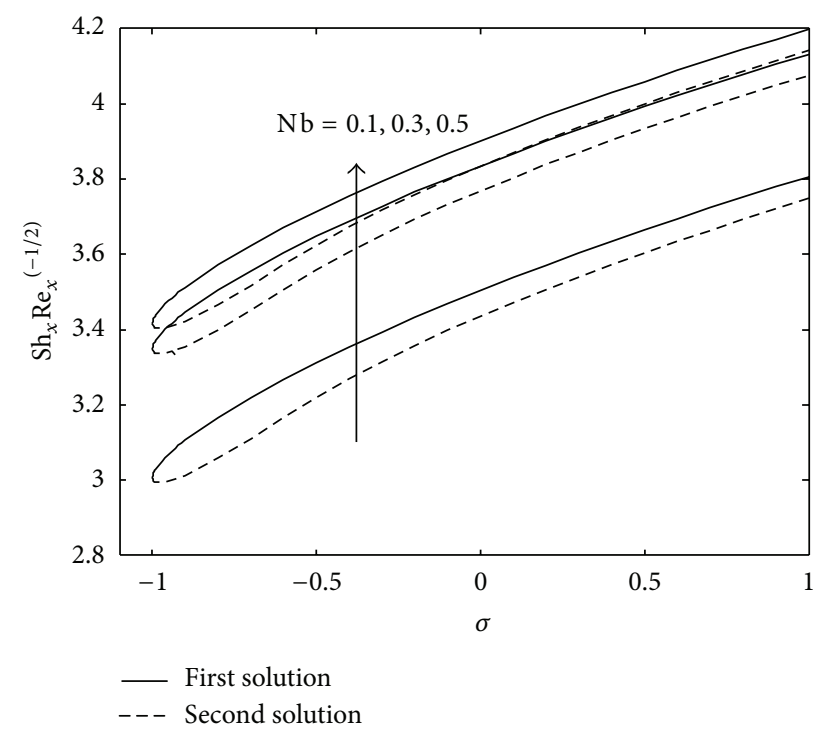

FIGURE 7: Variations of the local Sherwood number (concentration rates) with $\sigma$ for different values of $\mathrm{Nb}$ when $\mathrm{Nt}=0.5, \mathrm{Le}=2, \mathrm{Pr}=$ $6.8, \gamma=0.1$, and $S=2$.

Sherwood number were studied. Numerical solutions to the governing equations were obtained using a shooting method. The results for the local Nusselt number and the local Sherwood number are presented for different values of the governing parameters. The local Nusselt number decreases as the stretching/shrinking parameter increases. On the other hand, the local Sherwood number increases with the increasing of stretching/shrinking parameter. The local Nusselt number is consistently higher for higher values of the convective parameter but lower for higher values of the Brownian motion parameter and thermophoresis parameter. From the

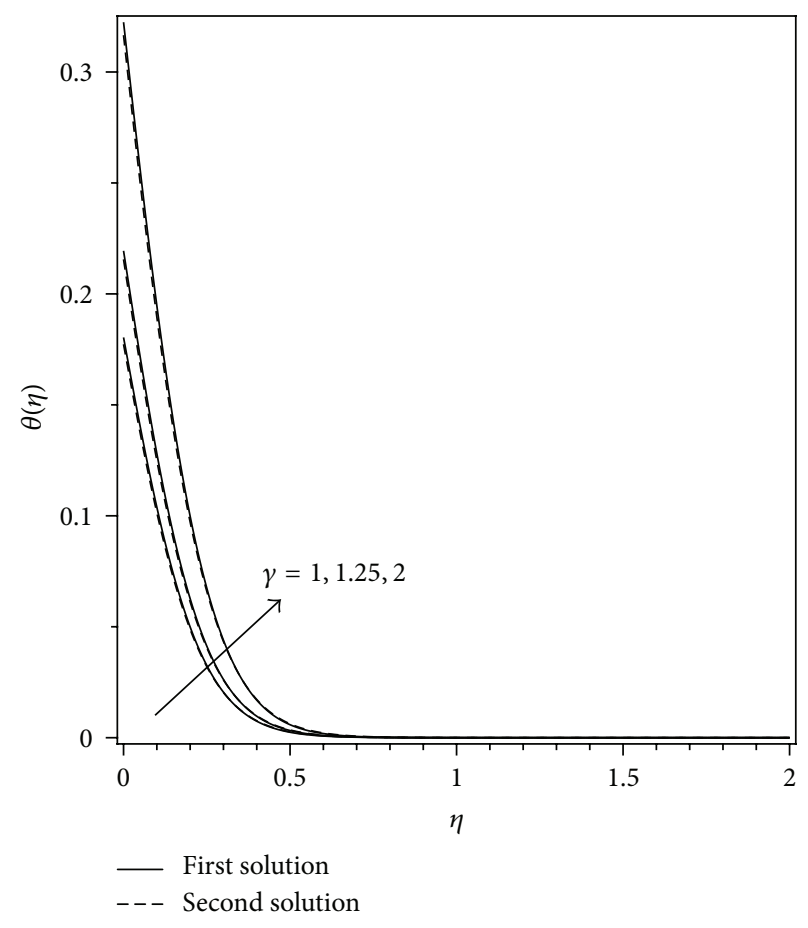

Figure 8: Temperature profiles for different values of $\gamma$ when $\mathrm{Nb}=$ $\mathrm{Nt}=0.5, \mathrm{Le}=2, \mathrm{Pr}=6.8, \sigma=-0.1$, and $S=2$.

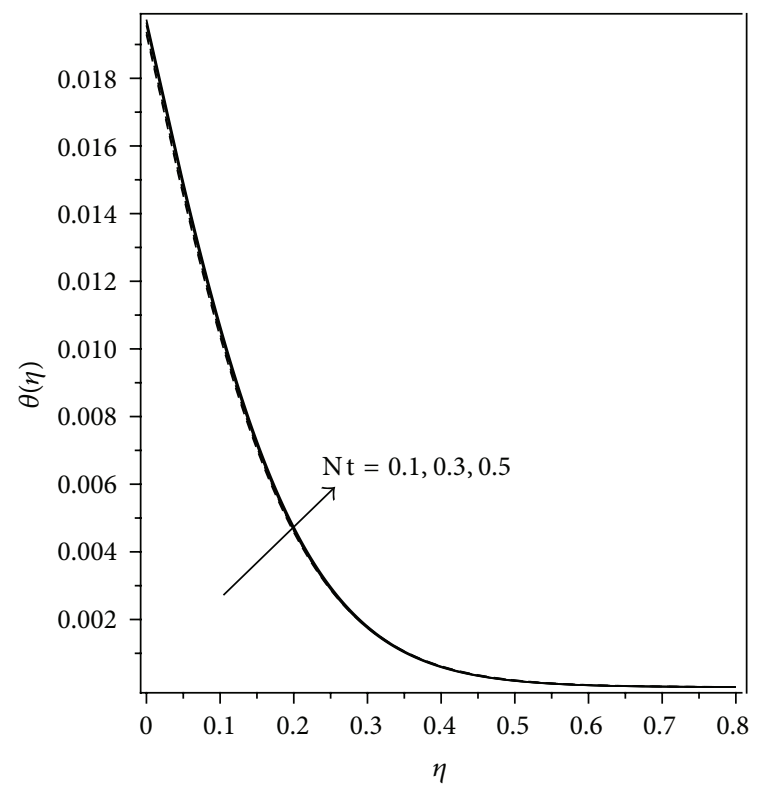

FIGURE 9: Temperature profiles for different values of $\mathrm{Nt}$ when $\mathrm{Nb}=$ $0.5, \mathrm{Le}=2, \operatorname{Pr}=6.8, \gamma=0.1, \sigma=-0.1$, and $S=2$.

results, Brownian motion parameter affects the surface heat transfer rate more than the thermophoresis parameter. The local Sherwood number increases with increasing Brownian motion parameter and decreases with increasing convective parameter and thermophoresis parameter. The results also indicate the existence of dual solutions for both stretching and shrinking cases. 


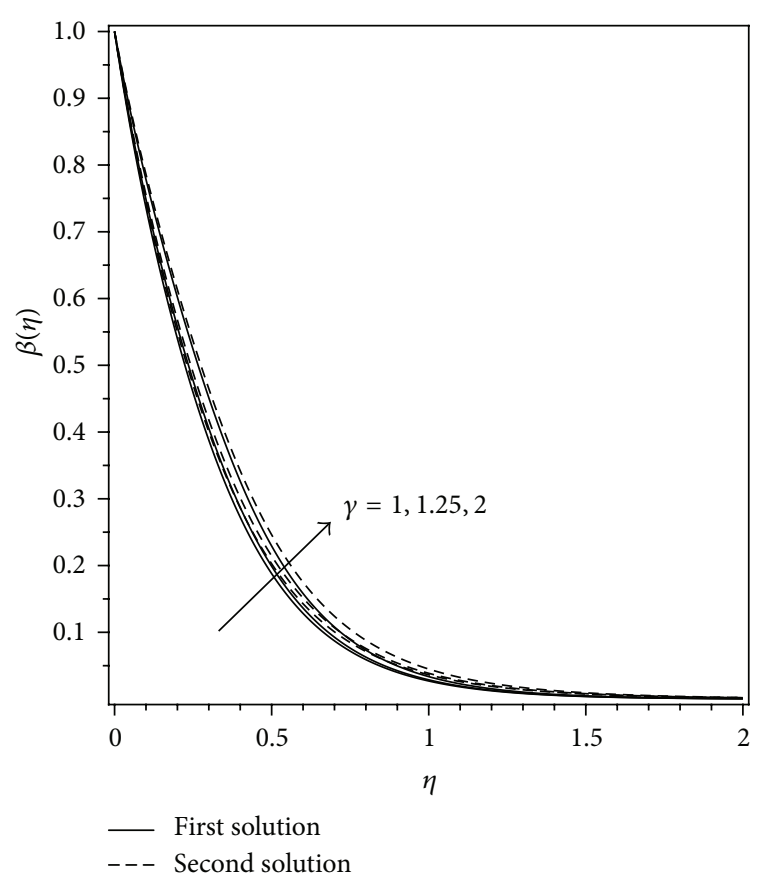

FIGURE 10: Concentration profiles for different values of $\gamma$ when $\mathrm{Nb}$ $=\mathrm{Nt}=0.5, \mathrm{Le}=2, \operatorname{Pr}=6.8, \sigma=-0.1$, and $S=2$.

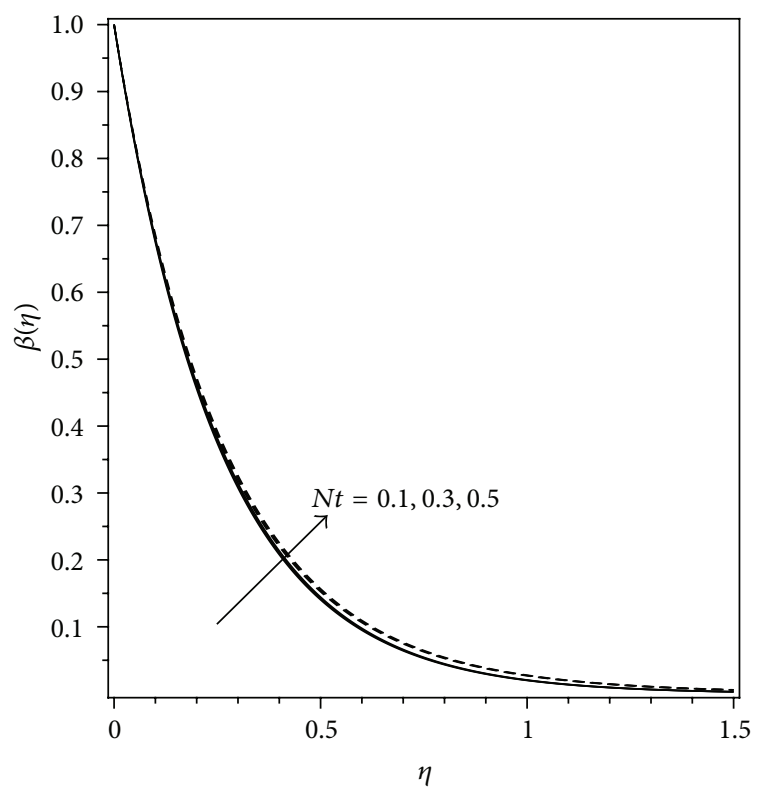

FIGURE 11: Concentration profiles for different values of $\mathrm{Nt}$ when $\mathrm{Nb}$ $=0.5, \mathrm{Le}=2, \operatorname{Pr}=6.8, \gamma=0.1, \sigma=-0.1$, and $S=2$.

\section{Conflict of Interests}

The authors declare that there is no conflict of interests regarding the publication of this paper.

\section{Acknowledgments}

The authors wish to express their thanks to the anonymous referees for their valuable comments and suggestions. The financial supports received from the Ministry of Higher Education, Malaysia (Project code: FRGS/1/2012/SG04/UKM/01/ 1), and the Universiti Kebangsaan Malaysia (Project code: DIP-2012-31) are gratefully acknowledged.

\section{References}

[1] E. G. Fischer, Extrusion of Plastics, Wiley, New York, NY, USA, 1976.

[2] L. J. Crane, "Flow past a stretching plate," Journal of Applied Mathematics and Physics, vol. 21, no. 4, pp. 645-647, 1970.

[3] C. Y. Wang, "Analysis of viscous flow due to a stretching sheet with surface slip and suction," Nonlinear Analysis: Real World Applications, vol. 10, no. 1, pp. 375-380, 2009.

[4] B. Sahoo, "Flow and heat transfer of a non-Newtonian fluid past a stretching sheet with partial slip," Communications in Nonlinear Science and Numerical Simulation, vol. 15, no. 3, pp. 602-615, 2010.

[5] M. Miklavčič and C. Y. Wang, "Viscous flow due to a shrinking sheet," Quarterly of Applied Mathematics, vol. 64, no. 2, pp. 283290, 2006.

[6] T. Fang, S. Yao, J. Zhang, and A. Aziz, "Viscous flow over a shrinking sheet with a second order slip flow model," Communications in Nonlinear Science and Numerical Simulation, vol. 15, no. 7, pp. 1831-1842, 2010.

[7] K. Bhattacharyya, S. Mukhopadhyay, and G. C. Layek, "Slip effects on boundary layer stagnation-point flow and heat transfer towards a shrinking sheet," International Journal of Heat and Mass Transfer, vol. 54, no. 1-3, pp. 308-313, 2011.

[8] S. U. S. Choi and J. A. Eastman, "Enhancing thermal conductivities of fluids with nanoparticles," in Proceedings of the ASME International Mechanical Engineering Congress and Exposition, San Francisco, Calif, USA, 1995.

[9] H. Masuda, A. Ebata, K. Teramae, and N. Hishinuma, "Alteration of thermal conductivity and viscosity of liquid by dispersing ultra-fine particles," Netsu Bussei, vol. 7, pp. 227-233, 1993.

[10] M. M. Rahman, M. A. Al-Lawatia, I. A. Eltayeb, and N. Al-Salti, "Hydromagnetic slip flow of water based nanofluids past a wedge with convective surface in the presence of heat generation (or) absorption," International Journal of Thermal Sciences, vol. 57, pp. 172-182, 2012.

[11] Y. Xuan and Q. Li, "Heat transfer enhancement of nanofluids," International Journal of Heat and Fluid Flow, vol. 21, no. 1, pp. 58-64, 2000

[12] Y. Xuan and W. Roetzel, "Conceptions for heat transfer correlation of nanofluids," International Journal of Heat and Mass Transfer, vol. 43, no. 19, pp. 3701-3707, 2000.

[13] S. Lee, S. U. S. Choi, S. Li, and J. A. Eastman, "Measuring thermal conductivity of fluids containing oxide nanoparticles," Journal of Heat Transfer, vol. 121, no. 2, pp. 280-288, 1999.

[14] R. K. Tiwari and M. K. Das, "Heat transfer augmentation in a two-sided lid-driven differentially heated square cavity utilizing nanofluids," International Journal of Heat and Mass Transfer, vol. 50, no. 9-10, pp. 2002-2018, 2007.

[15] J. Buongiorno, "Convective transport in nanofluids," Journal of Heat Transfer, vol. 128, no. 3, pp. 240-250, 2006.

[16] D. A. Nield and A. V. Kuznetsov, "The Cheng-Minkowycz problem for natural convective boundary-layer flow in a porous medium saturated by a nanofluid," International Journal of Heat and Mass Transfer, vol. 52, no. 25-26, pp. 5792-5795, 2009. 
[17] D. A. Nield and A. V. Kuznetsov, "Thermal instability in a porous medium layer saturated by a nanofluid," International Journal of Heat and Mass Transfer, vol. 52, no. 25-26, pp. 57965801, 2009.

[18] D. A. Nield and A. V. Kuznetsov, "The Cheng-Minkowycz problem for the double-diffusive natural convective boundary layer flow in a porous medium saturated by a nanofluid," International Journal of Heat and Mass Transfer, vol. 54, no. 1-3, pp. 374-378, 2011.

[19] A. V. Kuznetsov and D. A. Nield, "Natural convective boundarylayer flow of a nanofluid past a vertical plate," International Journal of Thermal Sciences, vol. 49, no. 2, pp. 243-247, 2010.

[20] A. V. Kuznetsov and D. A. Nield, "Double-diffusive natural convective boundary-layer flow of a nanofluid past a vertical plate," International Journal of Thermal Sciences, vol. 50, no. 5, pp. 712-717, 2011.

[21] W. A. Khan and I. Pop, "Boundary-layer flow of a nanofluid past a stretching sheet," International Journal of Heat and Mass Transfer, vol. 53, no. 11-12, pp. 2477-2483, 2010.

[22] N. Bachok, A. Ishak, and I. Pop, "Boundary-layer flow of nanofluids over a moving surface in a flowing fluid," International Journal of Thermal Sciences, vol. 49, no. 9, pp. 1663-1668, 2010.

[23] N. Bachok, A. Ishak, and I. Pop, "Unsteady boundary-layer flow and heat transfer of a nanofluid over a permeable stretching/shrinking sheet," International Journal of Heat and Mass Transfer, vol. 55, no. 7-8, pp. 2102-2109, 2012.

[24] W. A. Khan and A. Aziz, "Natural convection flow of a nanofluid over a vertical plate with uniform surface heat flux," International Journal of Thermal Sciences, vol. 50, no. 7, pp. 12071214, 2011.

[25] A. Aziz, "A similarity solution for laminar thermal boundary layer over a flat plate with a convective surface boundary condition," Communications in Nonlinear Science and Numerical Simulation, vol. 14, no. 4, pp. 1064-1068, 2009.

[26] A. Ishak, "Similarity solutions for flow and heat transfer over a permeable surface with convective boundary condition," Applied Mathematics and Computation, vol. 217, no. 2, pp. 837$842,2010$.

[27] O. D. Makinde and A. Aziz, "Boundary layer flow of a nanofluid past a stretching sheet with a convective boundary condition," International Journal of Thermal Sciences, vol. 50, no. 7, pp. 13261332, 2011.

[28] P. S. Gupta and A. S. Gupta, "Heat and mass transfer on a stretching sheet with suction or blowing," The Canadian Journal of Chemical Engineering, vol. 55, no. 6, pp. 744-746, 1977.

[29] A. Ishak, R. Nazar, and I. Pop, "Heat transfer over an unsteady stretching permeable surface with prescribed wall temperature," Nonlinear Analysis: Real World Applications, vol. 10, no. 5, pp. 2909-2913, 2009.

[30] A. Rasekh, D. D. Ganji, and S. Tavakoli, "Numerical solutions for a nanofluid past over a stretching circular cylinder with nonuniform heat source," Frontiers in Heat and Mass Transfer, vol. 3, pp. 1-6, 2012.

[31] J. H. Merkin, "On dual solutions occurring in mixed convection in a porous medium," Journal of Engineering Mathematics, vol. 20, no. 2, pp. 171-179, 1986.

[32] P. D. Weidman, D. G. Kubitschek, and A. M. J. Davis, "The effect of transpiration on self-similar boundary layer flow over moving surfaces," International Journal of Engineering Science, vol. 44, no. 11-12, pp. 730-737, 2006.
[33] A. Postelnicu and I. Pop, "Falkner-Skan boundary layer flow of a power-law fluid past a stretching wedge," Applied Mathematics and Computation, vol. 217, no. 9, pp. 4359-4368, 2011. 


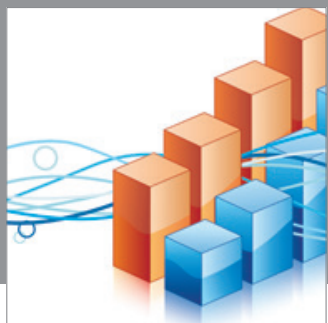

Advances in

Operations Research

mansans

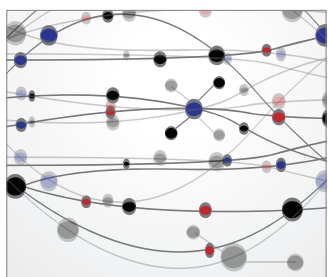

The Scientific World Journal
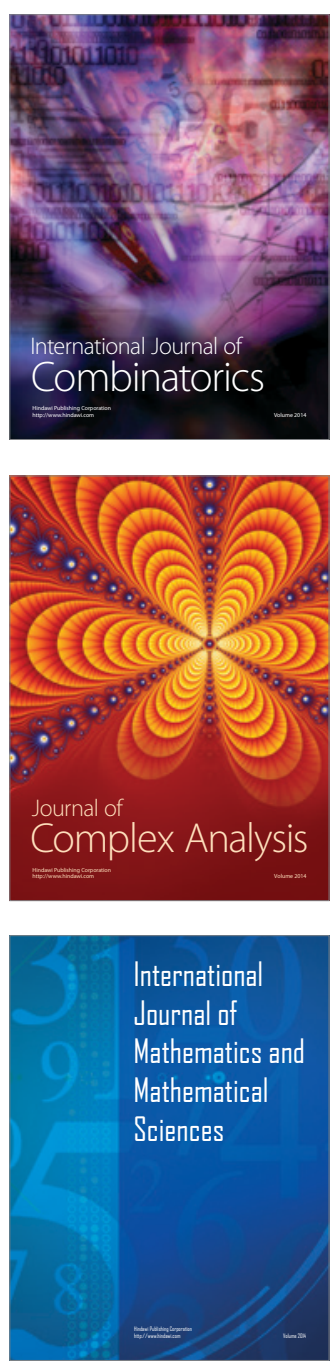
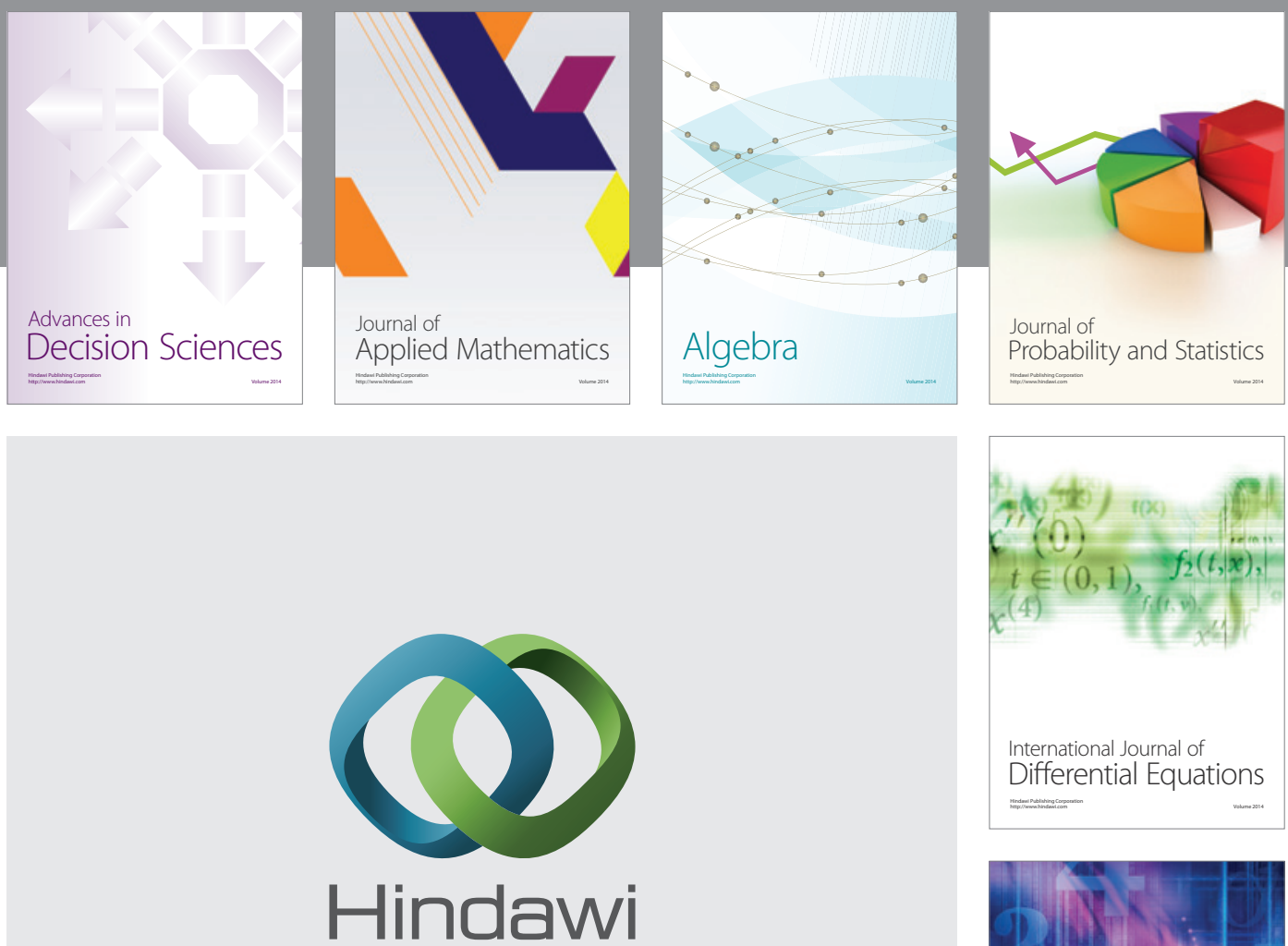

Submit your manuscripts at http://www.hindawi.com
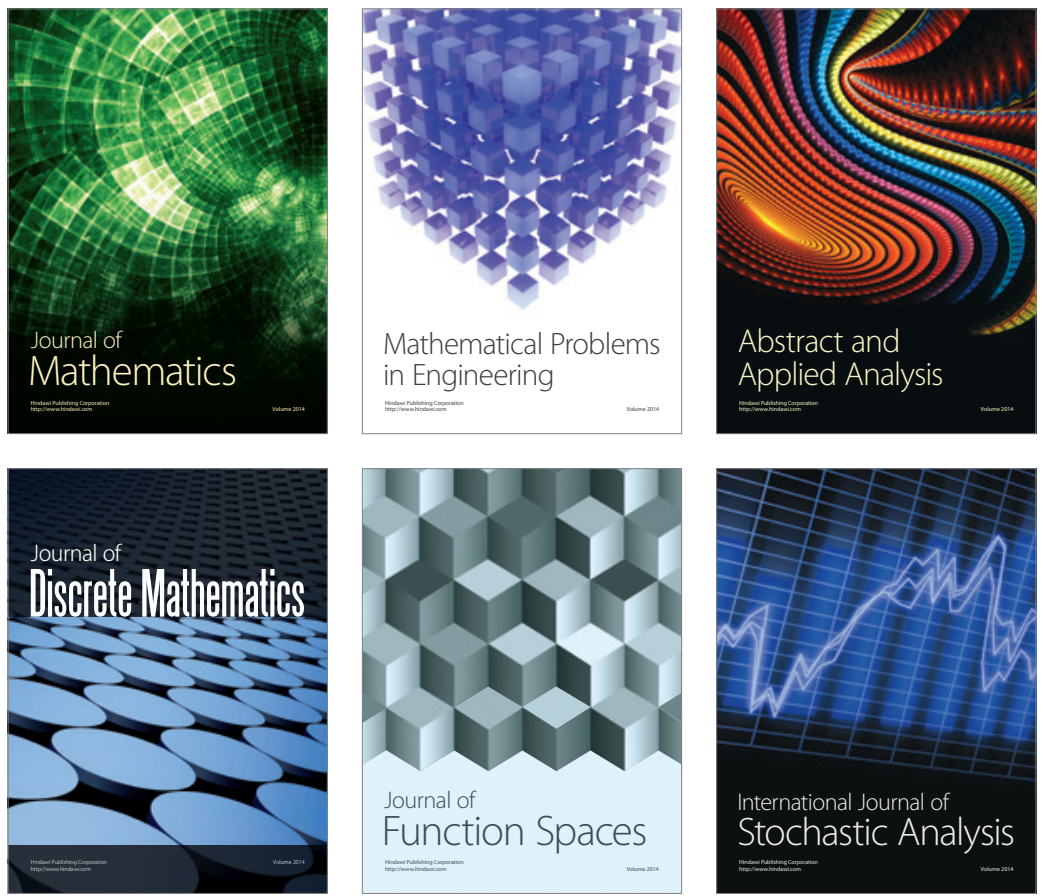

Journal of

Function Spaces

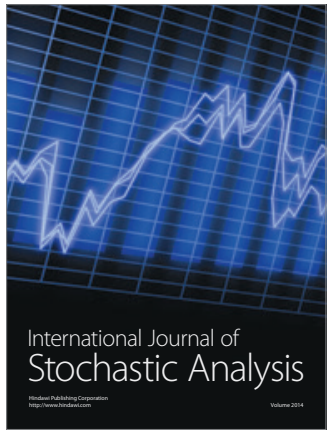

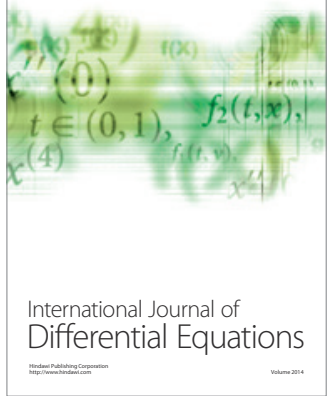
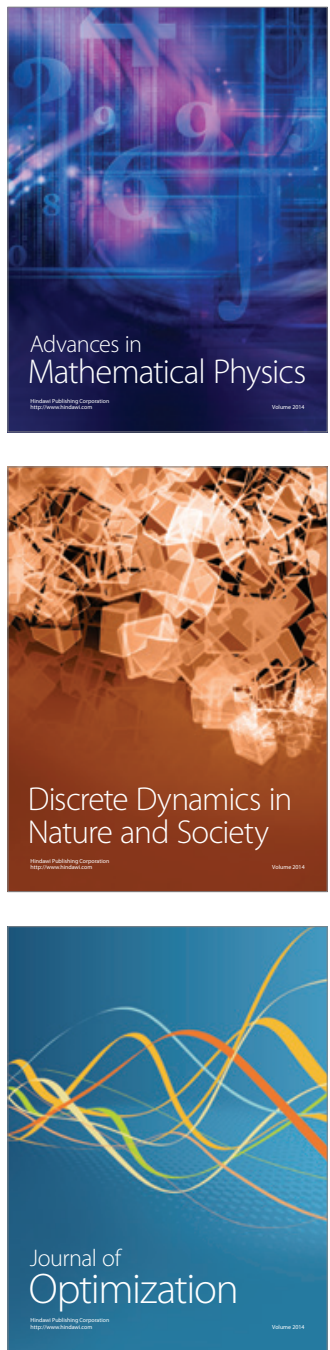